\title{
Between-network Functional Connectivity Is Modified by Age and Cognitive Task Domain
}

\author{
Eleanna Varangis ${ }^{1}$, Qolamreza Razlighi' ${ }^{1}$, Christian G. Habeck¹, \\ Zachary Fisher ${ }^{2}$, and Yaakov Stern ${ }^{1}$
}

\begin{abstract}
Research on the cognitive neuroscience of aging has identified myriad neurocognitive processes that are affected by the aging process, with a focus on identifying neural correlates of cognitive function in aging. This study aimed to test whether internetwork connectivity among six cognitive networks is sensitive to age-related changes in neural efficiency and cognitive functioning. A factor analytic connectivity approach was used to model network interactions during 11 cognitive tasks grouped into four primary cognitive domains: vocabulary, perceptual speed, fluid reasoning, and episodic memory. Results showed
\end{abstract}

\section{INTRODUCTION}

Studies of the cognitive neuroscience of aging have consistently found deleterious effects of aging on cognitive status and neural integrity and efficiency (Grady, 2008; Hedden \& Gabrieli, 2004). Over the course of adulthood, there exists a gradual decline in memory function, executive function, working memory, and attentional resources and a concomitant progression of neural degeneration, resulting in thinner cortex, white matter loss, and patterns of hyper- and hyporecruitment of brain regions. As such, a primary focus of research in this field has centered on identifying neural correlates of cognitive function in older adulthood. Recent studies have combined multiple behavioral and imaging modalities to investigate cognitive decline in aging and have found myriad neural metrics that may predict cognitive function in the context of aging (Hedden et al., 2016).

Recently neuroimaging research on cognitive aging has begun to utilize functional connectivity analyses to measure network-scale differences in neural recruitment between age groups. Of particular note is the emphasis placed on a neural network known as the default mode network (DMN), which is thought to be engaged primarily during rest and mind-wandering thought (Greicius, Supekar, Menon, \& Dougherty, 2009; Laird et al., 2009; Dosenbach et al., 2007; Esposito et al., 2006). Although this

${ }^{1}$ Columbia University, ${ }^{2}$ University of North Carolina at Chapel Hill

(C) 2018 Massachusetts Institute of Technology that both age and task domain were related to internetwork connectivity and that some of the connections among the networks were associated with performance on the in-scanner tasks. These findings demonstrate that internetwork connectivity among several cognitive networks is not only affected by aging and task demands but also shows a relationship with task performance. As such, future studies examining internetwork connectivity in aging should consider multiple networks and multiple task conditions to better measure dynamic patterns of network flexibility over the course of cognitive aging.

network has traditionally been studied during restingstate fMRI scans, recent studies have examined how this network is engaged during a cognitive task and have found that it "decouples" from task-related networks during performance of a task, such that correlated activity between a task-relevant network and the task-irrelevant DMN drops considerably in the presence of a task (Prakash, Heo, Voss, Patterson, \& Kramer, 2012; Sala-Llonch et al., 2012; Grady et al., 2010; Fox et al., 2005). This networkbased approach to fMRI analysis has gained traction within special populations, such as older adults (OAs) with and without neurodegenerative disease, as it allows examination of complex large-scale networks known to be structurally affected by brain aging and dementia (Fujiyama et al., 2016; Hirsiger et al., 2016; Jones et al., 2016; Liu et al., 2016; Suckling et al., 2015). Importantly, unlike younger adults, OAs do not show the same degree of anticorrelation between the DMN and task-specific networks, and this lack of anticorrelation between networks negatively correlates with performance on the task (Prakash et al., 2012; Miller et al., 2008). These findings suggest that this relatively greater connectivity between these two networks may underlie age-related differences in task performance.

Although this type of connectivity research has primarily focused on the DMN and executive function-related task networks (Damoiseaux, 2017; Prakash et al., 2012; Grady et al., 2010; Uddin, Kelly, Biswal, Castellanos, \& Milham, 2009), more recent studies have broadened their study of internetwork connectivity to examine network 
interactions across multiple cognitive networks. Specifically, interactions between the DMN and the dorsal attention network (DAN) have shown to be modulated by age and cognitive state (Esposito et al., 2018; Damoiseaux, 2017; Dixon et al., 2017; Amer, Anderson, Campbell, Hasher, \& Grady, 2016; Spreng, Stevens, Viviano, \& Schacter, 2016), and this interaction may be further coordinated by the frontoparietal control (FP) network (Avelar-Pereira, Bäckman, Wåhlin, Nyberg, \& Salami, 2017; Grady, Sarraf, Saverino, \& Campbell, 2016; Spreng, Sepulcre, Turner, Stevens, \& Schacter, 2013). In addition, another study found the salience (SAL) network to be critically involved in coordinating the DMN and central executive networks and found that this pattern was significantly disrupted in individuals with mild cognitive impairment (Chand, Wu, Hajjar, \& Qiu, 2017).

In this vein, several studies have attempted to expand this study of internetwork connectivity to the whole brain by examining system segregation or internetwork connectivity across many neural networks. Most of these studies have utilized resting-state fMRI data to show that OAs overall show greater between-network connections than younger adults (King et al., 2018; Ferreira et al., 2016; Geerligs, Renken, Saliasi, Maurits, \& Lorist, 2015; Chan, Park, Savalia, Petersen, \& Wig, 2014; Geerligs, Maurits, Renken, \& Lorist, 2014) and generally show less segregated/modular network organization (Geerligs et al., 2015; Song et al., 2014). Furthermore, some of these studies found a relationship between internetwork connectivity or segregation and performance on cognitive tasks (King et al., 2018; Chan et al., 2014; Geerligs et al., 2014), suggesting that this internetwork connectivity may underlie some of the variability in cognitive performance in the context of aging. However, although these largerscale network studies provide critical information about whole-brain network connectivity across the adult lifespan, only one of the studies mentioned above examined functional connectivity during performance of a cognitive task (Geerligs et al., 2014). Although some of the other studies make extensions to cognitive performance outside the scanner or relate resting-state connectivity data to BOLD activation during cognitive tasks (Chan, Alhazmi, Park, Savalia, \& Wig, 2017), none of the other studies examine internetwork connectivity across the whole brain during a cognitive challenge.

Thus, to bridge the findings from studies examining connectivity among a few networks during a cognitive task and those from studies examining internetwork connectivity across the whole brain at rest, this study examined between-network connectivity across six cognitive networks (DMN, DAN, FP, cingulo-opercular [CO], SAL, and memory) during 11 cognitive tasks corresponding to four primary cognitive domains (vocabulary, perceptual speed, fluid reasoning, and episodic memory). Based on prior studies showing reductions in network decoupling during cognitive task performance and generally greater connectivity between networks in aging, the hypotheses in this study were as follows: (1) Betweennetwork connectivity patterns among these predefined cognitive networks will be affected by both task type and participant age, and (2) connectivity between specific pairs of cognitive networks will account for significant variability in task performance. Because task relevance of networks will be in some ways determined by task domain, we expect patterns of internetwork connectivity to differ by domain. For example, although the DMN and the memory network may be irrelevant for FLUID and SPEED tasks, they may be relevant for VOCAB and MEM tasks, suggesting that patterns of interactions with these networks will likely be affected by task domain. On the other hand, networks such as the FP network and the CO network may be more broadly implicated in executive control and thus might be more generally relevant during all cognitive tasks. Furthermore, the SAL network has been implicated as one that might coordinate correlated activity between task-relevant and taskirrelevant networks, and thus, its task relevance may not be as likely to differ by task type.

Regarding Hypothesis 1, we expect that connectivity between task-relevant and task-irrelevant networks will be weaker than connectivity from task-relevant networks to other task-relevant networks. Because past studies on internetwork connectivity during a cognitive task have primarily used executive function tasks, these studies have found that task-relevant networks (i.e., FP, DAN) tend to show negative correlations with task-irrelevant networks (frequently the DMN) during a task and that aging disrupts this pattern (Esposito et al., 2018; AvelarPereira et al., 2017; Damoiseaux, 2017; Dixon et al., 2017; Amer et al., 2016; Grady et al., 2010, 2016; Spreng et al., 2013, 2016; Prakash et al., 2012; Sala-Llonch et al., 2012; Fox et al., 2005). As such, the task-specific hypotheses of this study are as follows: (1) During FLUID and SPEED tasks, connections between task-relevant (FP, DAN, CO) and task-irrelevant (DMN) will be reduced and connections among task-relevant networks will be increased; and (2) during VOCAB and MEM tasks, there will be fewer differences in connections between networks, because the DMN and the memory network (considered task-irrelevant in FLUID/SPEED tasks) may in these tasks be task relevant and implicated in mnemonic processing.

\section{METHODS}

\section{Sample}

The sample for this study came from participants who completed the baseline visit for the Reference Ability Neural Network study ( $n=426$; Stern et al., 2014). All participants were native English speakers, right-handed, free of MRI contraindications, and read at a fourth-grade reading level or above. Screening was performed before enrollment to ensure that no participants had any psychological or medical conditions that could affect cognitive 
function and that OAs did not meet criteria for dementia or mild cognitive impairment at baseline. Age was trichotomized to enable testing of moderation by age, resulting in three age groups: young adults (YAs; age = 20-39 years, $n=118$ ), middle-aged adults (MAs; age $=$ 40-60 years, $n=131$ ), and OAs (age $=61+$ years, $n=177$ ).

For the present analyses, the following additional inclusion criteria were established: completion of all 11 in-scanner tasks ( $n=338$; YA $n=96$, MA $n=110$, OA $n=132$ ) and less than $30 \%$ motion artifact data removal (scrubbing; Parkes, Fulcher, Yücel, \& Fornito, 2018; Power, Barnes, Snyder, Schlaggar, \& Petersen, 2012) within each of the four domain time series $(n=312$; YA $n=96$, MA $n=103$, OA $n=113$ ). Furthermore, one additional inclusion criteria concerned appropriate fit of the planned models and is detailed further alongside the analysis methodology described below. As a result, the balanced sample utilized in the analyses below was composed of 142 (YA $n=45$, MA $n=49$, OA $n=48$ ) healthy adults between the ages of 20 and $80(M=50.75, S D=17.335)$ who met all inclusion criteria.

\section{In-scanner Cognitive Tasks}

The cognitive variables included in this study are composed of their performance on tasks completed during the fMRI scan. The in-scanner tasks were designed to measure performance within each of the four reference abilities: vocabulary (VOCAB: synonyms and antonyms), perceptual speed (SPEED: digit symbol, letter comparison, pattern comparison), fluid reasoning (FLUID: paper folding, matrix reasoning, and letter sets), and episodic memory (MEM: logical memory, word order, and paired associates; for further information on tasks, please see Razlighi, Habeck, Barulli, \& Stern, 2017; Stern et al., 2014). In the vocabulary domain, the synonyms and antonyms tasks required participants to select a synonym/antonym (respectively) for a selected word from among four different options displayed on the same screen (15 trials per task). In the speed domain, the digit symbol task required participants to examine a digit symbol code table and determine whether a digit symbol pair on the sample screen was correct (90 trials), and the letter and pattern comparison tasks required participants to view a pair of strings of letters or figures composed of varying numbers of lines (respectively) presented simultaneously and indicate whether or not they were identical (60 trials per task). In the fluid domain, the paper-folding task required participants to select from five images one that represented the pattern of holes that would result from a set of folds in a piece of paper through which a hole is punched, the matrix reasoning task required participants to recognize a pattern from a series of pictures and identify the last missing piece of the pattern from among eight options, and the letter sets task required participants to identify which of a series of five sets of letters violated a rule expressed by the other four sets (7-18 self-paced trials for each FLUID task). In the memory domain, the logical memory task required participants to read a story one or two sentences at a time and then answer detailed fourchoice multiple-choice questions about the story (two stories, 10 questions per story), the word order task required participants to view a series of 12 words then later indicate which of four words immediately followed a probe word (10 probe trials), and the paired associates task required participants to view six pairs of words then indicate which of four options was originally paired with the probe word (two lists of pairs, six probe trials per list). One task, the picture-naming task from the VOCAB reference ability, was not included in the present analyses due to excessive in-scanner motion from participants speaking their responses aloud during the scanned task. Performance on VOCAB, FLUID, and MEM tasks is measured by the number of correct responses, whereas performance on SPEED tasks is measured by the average correct RT. To appropriately compare performance across these four domains, behavioral data were $z$-scored such that each domain score represents standardized deviation from the mean domain score of the entire set of participants-positive values represent behavioral performance (accuracy or RT) values above the normal mean, whereas negative values represent behavioral performance values below the normal mean. As such, for VOCAB, FLUID, and MEM tasks, positive $z$ scores reflect performance that is better (higher accuracy) than the mean, whereas for SPEED tasks, negative $z$ scores reflect performance that is better (faster) than the mean.

\section{fMRI Scan Parameters}

This study collected fMRI scans during the in-scanner tasks mentioned above. All participants completed these scans on a 3.0-T Philips Achieva Magnet over the course of two 2-hr MR imaging sessions. T1-weighted images of the whole brain were acquired for each participant with a magnetization prepared rapid gradient-echo sequence with the following parameters: echo time/ repetition time $=3 / 6.5 \mathrm{msec}$, field of view $=256 \mathrm{~mm}$, flip angle $=8^{\circ}$, in-plane resolution $=256 \times 256$ voxels, slice thickness $/$ gap $=1 / 0 \mathrm{~mm}$, slices $=180$. fMRI BOLD scans were collected during each of the 11 in-scanner tasks mentioned above with the following parameters: echo time/repetition time $=20 / 2000 \mathrm{msec}$; field of view $=$ $240 \mathrm{~mm}$; flip angle $=72^{\circ}$; in-plane resolution $=112 \times$ 112 voxels; slice thickness $/$ gap $=3 / 0 \mathrm{~mm}$; slices $=41$.

\section{fMRI Data Processing}

Images were preprocessed using an in-house developed native space method (Razlighi et al., 2014). Briefly, slicetiming correction was performed using the FSL slicetimer tool. We then used MCFLIRT (motion correction tool in the FSL package; Jenkinson, Beckmann, Behrens, Woolrich, \& Smith, 2012) to register all volumes to a 
reference image (Jenkinson, Bannister, Brady, \& Smith, 2002). The reference image was generated by registering ( $6 \mathrm{df}, 256$ bins mutual information, and sinc interpolation) all volumes to the middle volume and averaging them. We then used the method described in Power et al. (2012) to calculate framewise displacement (FWD) from the six motion parameters and root mean square difference (RMSD) of the BOLD percentage signal in the consecutive volumes for each participant. To be conservative, we lowered the threshold of our RMSD to $0.3 \%$ (it was originally suggested to be $0.5 \%$ ). RMSD was computed on the motion-corrected volumes before temporal filtering. The contaminated volumes were detected by the criteria FWD $>0.5 \mathrm{~mm}$ or RMSD $>0.3 \%$. Identified contaminated volumes were replaced with new volumes generated by linear interpolation of adjacent volumes. Volume replacement was done before bandpass filtering (Carp, 2013). The motion-corrected signals were passed through a bandpass filter with the cutoff frequencies of 0.01 and $0.09 \mathrm{~Hz}$. We used flsmathsbptf to do the filtering in this study (Jenkinson et al., 2012). Finally, we residualized the motion-corrected, scrubbed, and temporally filtered volumes by regressing out the FWD, RMSD, left and right hemisphere white matter, and lateral ventricular signals (Birn, Diamond, Smith, \& Bandettini, 2006).

T1 image segmentation was done using FreeSurfer (Fischl et al., 2002, 2004; Dale, Fischl, \& Sereno, 1999) and visually checked for any inaccuracies. In the event that we observed any inaccuracy in the FreeSurfer segmentation, we made corrections using the FreeSurfer provided guidelines for troubleshooting data. The coordinates of the 264 putative functional nodes derived from a brainwide graph described by Power and colleagues (2011) were then transferred to each participant's T1 space via nonlinear registration of the participant's structural scan to the Montreal Neurological Institute template using the ANTS software package. A spherical mask with 10-mm radius, centered at each transferred coordinate, was generated and intersected with the FreeSurfer gray matter mask to obtain the ROI mask for the 264 functional nodes. An intermodal, intraparticipant, rigid body registration of fMRI reference image and T1 scan was performed with FLIRT with $6 d f$, normalized mutual information as the cost function (Jenkinson \& Smith, 2001), and used to transfer all ROI masks from T1 space to fMRI space. These transferred ROI masks were used to average all the voxels within each mask to obtain a single fMRI time series for each node.

Functional connectivity time-series data were then extracted from each of the 264 coordinate-based ROIs within each participant's preprocessed fMRI task scans. Six networks were preselected for analysis based on their role in cognitive processes identified in previous studies and as outlined by the Power et al. (2011) coordinate system: FP network (25 ROIs), CO network (14 ROIs), DAN (11 ROIs), memory network (MEM; 5 ROIs), SAL network (18 ROIs), and DMN (58 ROIs). Time-series data were then concatenated by domain, yielding four sets of time-series data that were modeled separately as blocked designs: VOCAB (concatenated synonyms and antonyms data; 388 volumes), SPEED (concatenated digit symbol, letter comparison, and pattern comparison data; $595 \mathrm{vol}-$ umes), FLUID (concatenated paper folding, matrix reasoning, and letter sets data; 1290 volumes), and MEM (concatenated logical memory, word order, and paired associates data; 517 volumes).

\section{Analytic Methodology}

To model network-level time-series interactions, a factor analytic functional connectivity methodology was utilized. Data were analyzed in $\mathrm{R}$ using a structural equation modeling approach, model-implied instrumental variable estimation (Bollen, 1996, 2001), appropriate for highdimensional data (MIIVsem; https://cran.r-project.org/ package $=$ MIIVsem; Fisher, Bollen, Gates, \& Rönkkö, 2017). This approach is designed for robust estimation of high-dimensional structural equation models and has recently been extended to handle individual-level multivariate time-series data (Fisher, Bollen, \& Gates, in press). Furthermore, this approach allows for the estimation of variance and covariance parameters of network time series along with bootstrap standard errors. Each individual's ROI-level time-series data from the 131 ROIs reflecting the networks selected above was loaded onto the corresponding predefined network latent factors, resulting in a six-factor model estimated at the participant level, where the primary outcome measures were correlations between the six network factors (see Figure 1). This approach is not only a robust technique for modeling repeated measures at the participant level but also represents a more flexible method for modeling network-level time-series data in that it does not impose group-level assumptions on the contributions of each ROI with respect to network function. Thus, ROIs are allowed to freely load on the latent network factors, allowing participants to differ in the respective contributions of each ROI in the predefined networks and reducing the effect of any outliers on network time-series estimation. This analysis was performed separately for each cognitive domain of interest: VOCAB, SPEED, FLUID, and MEM, resulting in four correlation matrices of size $6 \times 6$ for each participant. Individual-level correlation coefficients between latent network factors for each of the four domains were then $z$-transformed and exported to SPSS for MANOVA and correlational analyses. The additional inclusion criterion referenced above was valid estimation of the prespecified factor structure: To be included in the analyses, each participant had to have valid data for the estimation of the factor structure and correlations among factors. Although variability in network architecture that deviates from the prespecified criteria may be critical to examine, it 


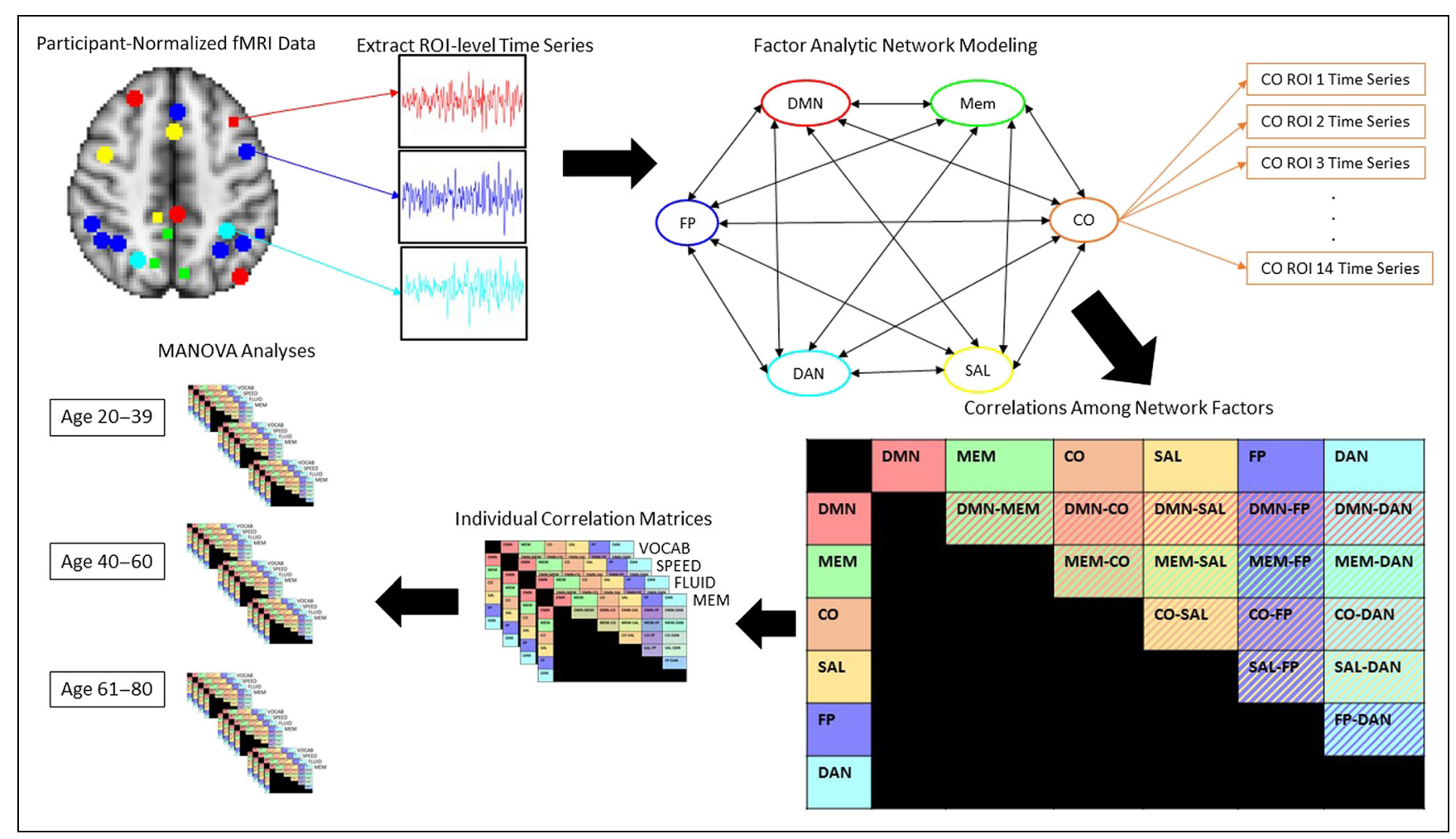

Figure 1. Factor analytic connectivity analysis pipeline.

was beyond the scope of the present analyses, and thus, only participants whose network structure fit the structure that was prespecified based on empirically defined network structures were included. Thus, after the data were exported to SPSS, all participants who had errors related to computation of latent factor variances during factor structure estimation were removed to ensure that only participants with valid estimation of the proposed factor structure were included in the analyses.

To explore the effects of specific connection (within participants: i.e., FP-DMN), cognitive domain (within participants: VOCAB, SPEED, FLUID, and MEM), age (between participants: YA, MA, and OA), and their interactions on internetwork correlations, multivariate analyses of variance were conducted. Follow-up Spearman correlational analyses were then conducted to test for any relationship between internetwork connectivity and task performance. Because of the exploratory nature of this analysis, a Spearman correlational approach was utilized over a Pearson correlational approach to test for robust monotonic relationships between connectivity and task performance without making any assumptions about the linearity of this relationship (i.e., Geerligs et al., 2014, 2015). In the first set of correlational analyses, Spearman correlational tests were conducted across the entire age range. Next, similar to previous studies assessing the relationship between functional connectivity and cognitive/behavioral outcomes (Ferreira et al., 2016; Geerligs et al., 2015; Chan et al., 2014), partial Spearman correlational tests were conducted with respect to age to remove any pure effect of age on both task performance and functional connectivity. Finally, Spearman correlational tests were conducted within each of the three age groups to examine whether these relationships remained stable across the three age groups.

\section{RESULTS}

\section{Participants}

Demographic characteristics of participants in this study are summarized in Table 1 . To test whether this subsample of the data set was in any way biased, independent sample $t$ tests were performed, ensuring no group differences between excluded and included participants on age $(p=.091)$, education $(p=.311)$, sex $(p=$ $.820)$, race $(p=.168)$, ethnicity $(p=.716)$, National Adult Reading Test (NART) IQ $(p=.070)$, and SPEED $(p=.124)$ and MEM $(p=.265)$ tasks; however, included participants tended to have higher scores on VOCAB $(p=.040)$ and FLUID $(p=.008)$ tasks. Furthermore, chi-square tests were run for each inclusion/exclusion step to ensure that exclusion of participants was not biased by age group. Chi-square tests for each exclusion step showed that, at enrollment, there were more OAs than MAs or YAs $(p=.001)$ and more OAs were excluded for not having balanced fMRI task data $(p=$ $.002)$ and greater than $30 \%$ scrubbing $(p<.001)$; however, there was no bias in the number of participants excluded for improper estimation of factor structure 
Table 1. Demographic Characteristics of the Sample by Age Group: YA (20-39 years), MA (40-60 years), and OA (61-80 years)

\begin{tabular}{|c|c|c|c|c|c|}
\hline & $Y A$ & $M A$ & $O A$ & Overall & Test Statistic $(p)$ \\
\hline$n$ & 45 & 49 & 48 & 142 & $\mathrm{n} / \mathrm{a}$ \\
\hline Age $(S D)$ & $29.73(5.127)$ & $50.69(5.864)$ & $70.50(4.524)$ & $50.75(17.335)$ & $F(2,139)=711.735(<.001)^{\mathrm{a}}$ \\
\hline Education $(S D)$ & $16.16(1.731)$ & $15.71(1.947)$ & $16.90(2.769)$ & $16.25(2.242)$ & $F(2,139)=3.554(.031)^{b}$ \\
\hline NART IQ $(S D)$ & 114.81 (8.279) & $115.93(8.301)$ & $121.66(6.222)$ & $117.54(8.165)$ & $F(2,134)=10.598(<.001)^{c}$ \\
\hline$\%$ Female & 61.4 & 49.0 & 57.5 & 55.7 & $\chi_{2}^{2}=1.527(.466)$ \\
\hline $\operatorname{VOCAB}(S D)$ & $-0.252(0.846)$ & $0.146(0.122)$ & $0.466(0.792)$ & $0.126(0.875)$ & $F(2,138)=8.582(<.001)^{\mathrm{d}}$ \\
\hline SPEED $(S D)$ & $-0.660(0.690)$ & $-0.043(0.712)$ & $0.371(0.748)$ & $-0.085(0.825)$ & $F(2,134)=22.713(<.001)^{a}$ \\
\hline FLUID $(S D)$ & $0.480(0.740)$ & $0.093(0.748)$ & $-0.053(0.740)$ & $0.166(0.770)$ & $F(2,136)=6.198(.003)^{\mathrm{e}}$ \\
\hline MEM $(S D)$ & $0.406(0.667)$ & $0.053(0.594)$ & $-0.239(0.631)$ & $0.068(0.678)$ & $F(2,135)=11.813(<.001)^{\mathrm{e}}$ \\
\hline
\end{tabular}

Bolded test statistics indicate those that are significant, and bolded group level means highlight values that significantly differ by group.

${ }^{a}$ All three groups significantly differ $(p<.05$; Tukey Honestly Significant Difference [HSD]).

${ }^{\mathrm{b}} \mathrm{OA}>\mathrm{MA}(p<.05 ;$ Tukey HSD $)$.

${ }^{\mathrm{c}} \mathrm{OA}>\mathrm{MA}$ and YA $(p<.05$; Tukey HSD $)$.

${ }^{\mathrm{d}} \mathrm{OA}>\mathrm{YA}$.

${ }^{\mathrm{e}} \mathrm{YA}>\mathrm{MA}$ and OA $(p<.05$; Tukey HSD $)$.

$(p=.383)$. Thus, more OAs were excluded for not completing all 11 in-scanner tasks and for having more data scrubbed; however, participant dropout was similar for proper estimation of factor structure.

Furthermore, to examine this effect of age on scrubbing across all 338 participants with balanced fMRI task data, a 3 (Age group: YA, MA, OA) $\times 4$ (task Domain: VOCAB, SPEED, FLUID, MEM) MANOVA was used to probe the effects of age and domain on percent scrubbing. Results showed that there was a main effect of Age, $F(2,335)=14.081, p<.001$, and a main effect of Domain, $F(3,1005)=18.595, p<.001$, but no interaction between Domain and Age, $F(6,1005)=1.646, p=.131$. The main effect of Age showed that OAs $(M=9.530 \%)$ had more data scrubbed than both younger $(M=3.741 \%$, $p<.001)$ and middle-aged $(M=5.906 \%, p=.003)$ participants. Furthermore, more older $(n=19)$ and middleaged $(n=7)$ adults were excluded for having greater than $30 \%$ scrubbing on any given task domain than younger adults $(n=0)$. The main effect of Domain showed that FLUID ( $M=8.420 \%)$ tasks had more data scrubbed than $\operatorname{VOCAB}(M=5.762 \%, p<.001)$, SPEED $(M=$ $5.990 \%, p<.001)$, or MEM $(M=6.655 \%, p<.001)$ tasks. However, of note is that FLUID tasks were the longest task scans of the two scan sessions, so this greater proportional amount of motion during these tasks could be partly due to longer acquisition times.

\section{MANOVA Results}

Internetwork $z$-transformed correlation values were analyzed in a MANOVA to assess the independent and interac- tive effects of participant Age (between participants), task Domain (within participants), and Network pairing (within participants) on internetwork correlations during performance of the in-scanner tasks. Thus, the primary interactions of interest were between age and network pairing, domain and network pairing, and the three-way interaction, as these would suggest that between-network correlations are affected by participant Age, task Domain, or both. Means for each connection for the aforementioned two-way interactions are depicted in Figures 2 (Age $\times$ Connection) and 3 (Domain $\times$ Connection) and represent summaries of the data used for the MANOVA analyses reported below. The values depicted are the mean $z$-transformed correlation between two specific networks for each age group (Figure 2) or task domain (Figure 3).

A 3 (Age group: YA, MA, and OA) $\times 4$ (Domain: VOCAB, SPEED, FLUID, and MEM) $\times 15$ (internetwork Connection) MANOVA revealed a significant main effect of Domain, $F(3,417)=8.577, p<.001$, a main effect of Connection, $F(14,1946)=84.563, p<.001$, an interaction between Connection and Age group, $F(28,1946)=$ $4.024, p<.001$, and an interaction between Domain and Connection, $F(42,5838)=12.309, p<.001$. The main effect of Age, $F(2,139)=0.116, p=.891$, and the interactions between Domain and Age group, $F(6,417)=0.626$, $p=.710$, and Domain, Connection, and Age group, $F(84$, $5754)=1.185, p=.120$, were not significant. Post hoc analyses of the interactions between connection and age (see Table 2; Figure 2), and connection and domain (see Table 3; Figure 3) showed a significant effect of age and domain on internetwork connection strength across many internetwork connections. In the interaction 
Figure 2. Between-network $z$-transformed correlation coefficients for the interaction between age and connection. Asterisks represent significance of age difference at each connection.

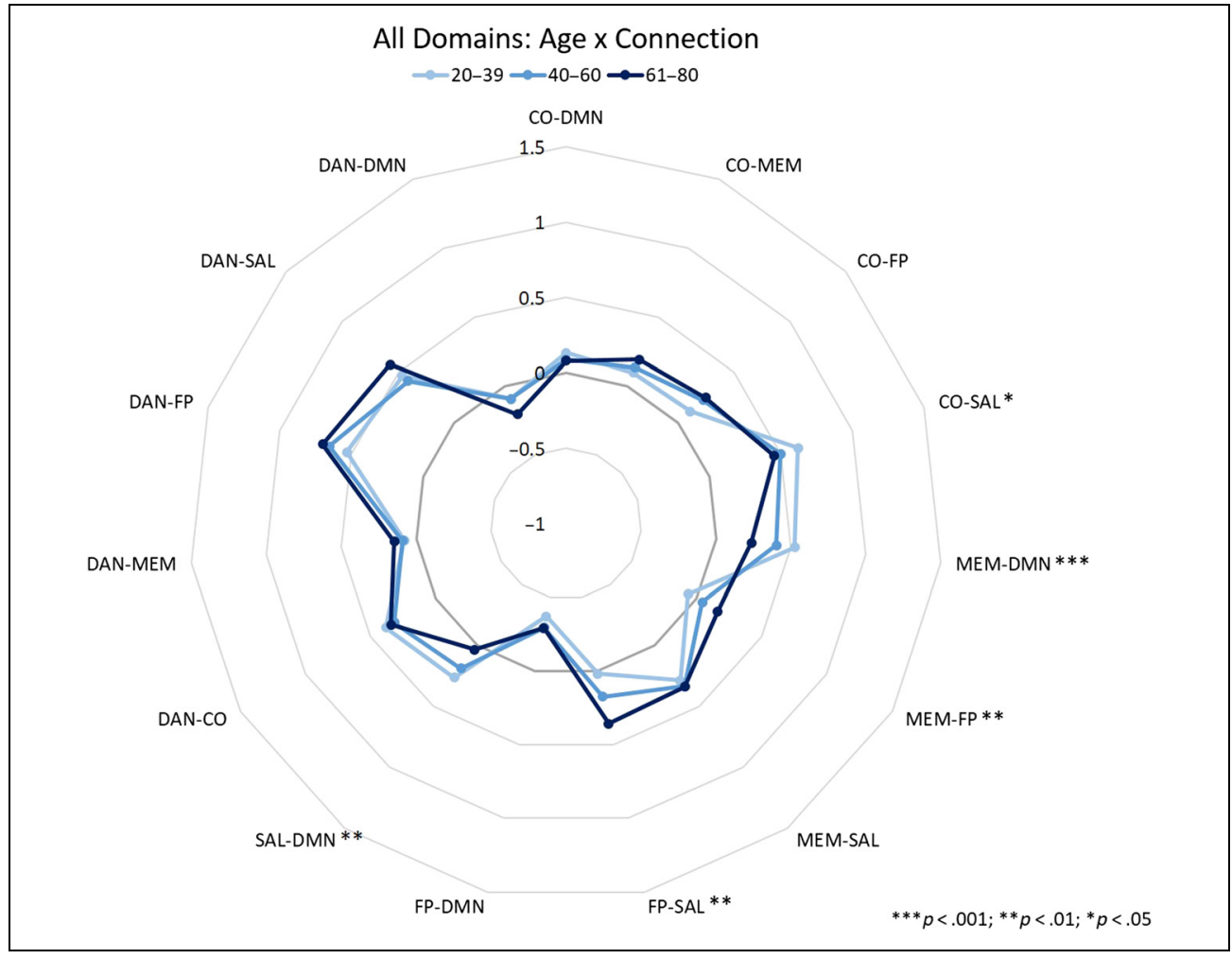

between age and connection, OAs showed greater correlations between MEM-FP and FP-SAL than YAs, whereas YAs showed greater correlations between CO-SAL, MEMDMN, and SAL-DMN than OAs (see Table 2; Figure 2). In the interaction between domain and connection, only two connections did not show a significant effect of domain on the correlations between networks: MEM-SAL and SAL-DMN. The 13 remaining connections showed some alteration in correlation strength, depending on the task domain-for example, the DAN-FP connection
Figure 3. Between-network $z$-transformed correlation coefficients for the interaction between task domain and connection. Asterisks represent significance of domain difference at each connection.

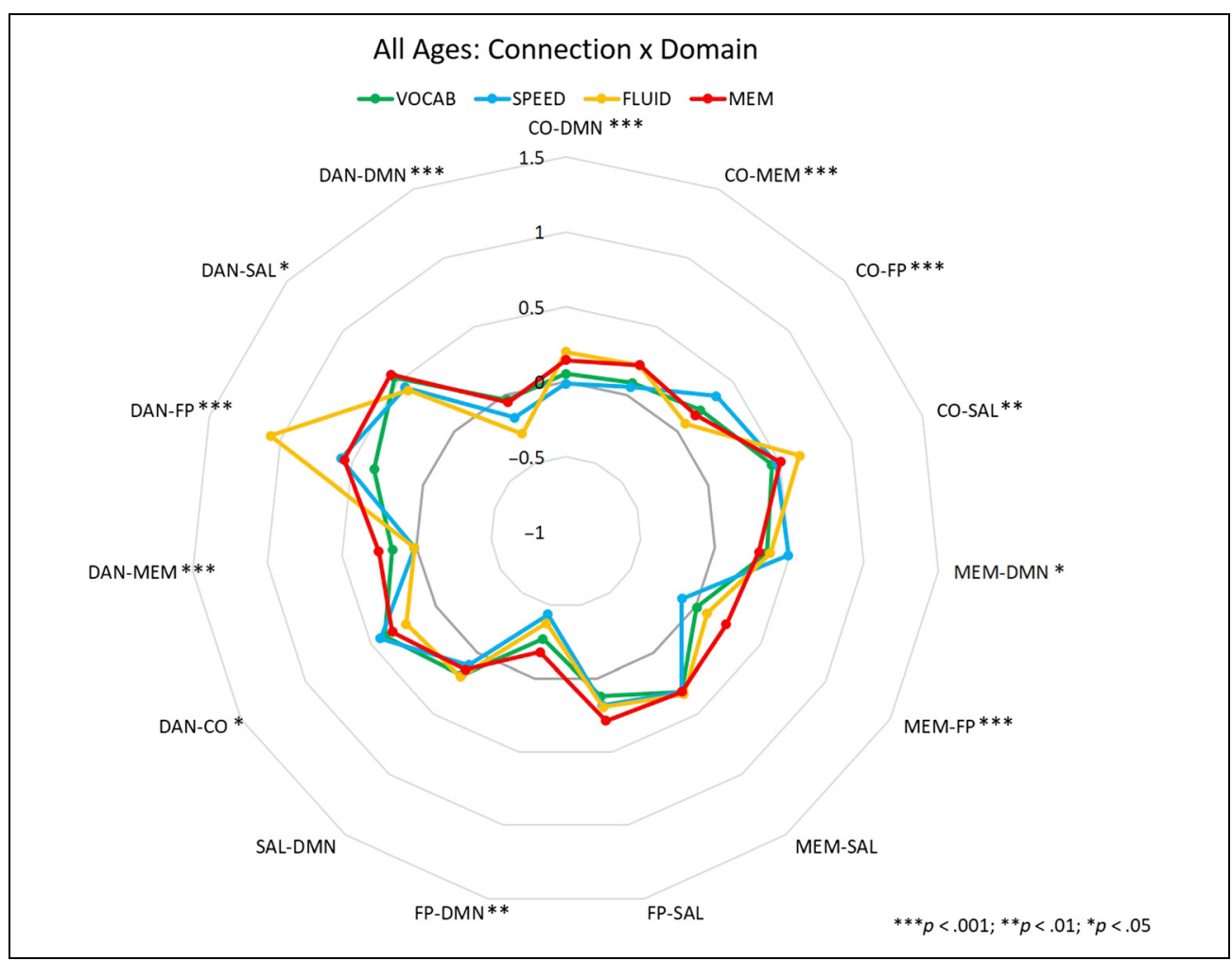


Table 2. MANOVA Results for Post hoc Analyses of the Interaction between Age Group (YA, MA, and OA) and Connection $(d f$ between $=2, d f$ within $=139$ for All Statistics Reported $)$

\begin{tabular}{|c|c|c|c|c|c|}
\hline Connection & $F$ & $p$ & YA vs. $M A$ & $Y A$ vs. $O A$ & MA vs. $O A$ \\
\hline CO-DMN & 0.202 & .817 & $\mathrm{n} / \mathrm{a}$ & $\mathrm{n} / \mathrm{a}$ & $\mathrm{n} / \mathrm{a}$ \\
\hline CO-MEM & 2.635 & .075 & $\mathrm{n} / \mathrm{a}$ & $\mathrm{n} / \mathrm{a}$ & $\mathrm{n} / \mathrm{a}$ \\
\hline CO-FP & 1.685 & .189 & $\mathrm{n} / \mathrm{a}$ & $\mathrm{n} / \mathrm{a}$ & $\mathrm{n} / \mathrm{a}$ \\
\hline CO-SAL & 3.119 & .047 & $0.131(.277)$ & $0.212(.038)$ & $0.081(.597)$ \\
\hline MEM-DMN & 9.671 & $<.001$ & $0.124(.251)$ & $0.337(<.001)$ & $0.214(.016)$ \\
\hline MEM-FP & 7.351 & .001 & $-0.044(.764)$ & $-0.228(.001)$ & $-0.184(.010)$ \\
\hline MEM-SAL & 0.737 & .481 & $\mathrm{n} / \mathrm{a}$ & $\mathrm{n} / \mathrm{a}$ & $\mathrm{n} / \mathrm{a}$ \\
\hline FP-SAL & 5.742 & .004 & $-0.133(.312)$ & $-0.309(.003)$ & $-0.176(.126)$ \\
\hline FP-DMN & 0.472 & .625 & $\mathrm{n} / \mathrm{a}$ & $\mathrm{n} / \mathrm{a}$ & $\mathrm{n} / \mathrm{a}$ \\
\hline SAL-DMN & 5.914 & .003 & $0.119(.344)$ & $0.292(.002)$ & $0.173(.101)$ \\
\hline DAN-CO & 0.458 & .634 & $\mathrm{n} / \mathrm{a}$ & $\mathrm{n} / \mathrm{a}$ & $\mathrm{n} / \mathrm{a}$ \\
\hline DAN-MEM & 0.798 & .452 & $\mathrm{n} / \mathrm{a}$ & $\mathrm{n} / \mathrm{a}$ & $\mathrm{n} / \mathrm{a}$ \\
\hline DAN-FP & 1.767 & .175 & $\mathrm{n} / \mathrm{a}$ & $\mathrm{n} / \mathrm{a}$ & $\mathrm{n} / \mathrm{a}$ \\
\hline DAN-SAL & 2.720 & .069 & $\mathrm{n} / \mathrm{a}$ & $\mathrm{n} / \mathrm{a}$ & $\mathrm{n} / \mathrm{a}$ \\
\hline DAN-DMN & 1.201 & .304 & $\mathrm{n} / \mathrm{a}$ & $\mathrm{n} / \mathrm{a}$ & $\mathrm{n} / \mathrm{a}$ \\
\hline
\end{tabular}

Contrasts are represented in columns $4-6$ by the mean difference in $z$-transformed correlation coefficients between groups ( $p$ value in parentheses). Bolded values reflect mean differences that are statistically significant.

showed significantly greater connectivity during tasks of fluid reasoning than tasks from any other domains (and greater connectivity during memory tasks than vocabulary tasks; see Table 3; Figure 3).

\section{Task Performance Correlations}

To assess any relationships between task performance and internetwork connectivity during the task, Spearman correlational analyses were conducted. Full correlation tables are presented for each of the three correlational analyses presented here (see Tables 4-6); however, only results that survive multiple comparisons correction using the Benjamini-Hochberg procedure across all correlations analyzed (60 per analysis- 15 connections $\times 4$ domains) are discussed here. In the first set of results, Spearman correlational tests were conducted across the entire age range. Results showed that FLUID task performance was negatively correlated with DANMEM connectivity, such that better performance on FLUID tasks was associated with a weaker correlation between the latent factors reflecting the DAN and MEM networks (see Table 4; $r_{139}=-.312, p<.001$ ). In the second set of results, partial Spearman correlational tests were conducted with respect to age to remove any pure effect of age on both task performance and functional connectivity. After removing the effect of age from these relationships, the correlation between FLUID perfor- mance and DAN-MEM connectivity remained significant (see Table $5 ; r_{139}=-.285, p=.001$ ). In the third set of results, Spearman correlational tests were conducted within each age group to examine whether these relationships remained stable across the three age groups. Results from this analysis showed that, across the three age groups, the only correlation that survived false discovery rate correction was a negative relationship between VOCAB task performance and FP-DMN connectivity in YAs (see Table $6 ; r_{45}=-.483, p=.001$ ).

\section{DISCUSSION}

Results from this study show that patterns of internetwork connectivity during task performance are modulated by age and task domain. Specifically, age may particularly affect connectivity directed from the memory and SAL networks, such that OAs show greater connectivity between the memory network and a task-relevant network (FP), altered connectivity directed from the SAL network to task-relevant networks (CO and FP), and reduced connectivity from memory and SAL networks to the DMN. However, the present set of results did not replicate findings from previous studies showing reduced anticorrelation between the DMN and the FP network in OAs (Prakash et al., 2012; Miller et al., 2008)—in the interaction between age and connection, there was no effect of age on connectivity between these two networks. 


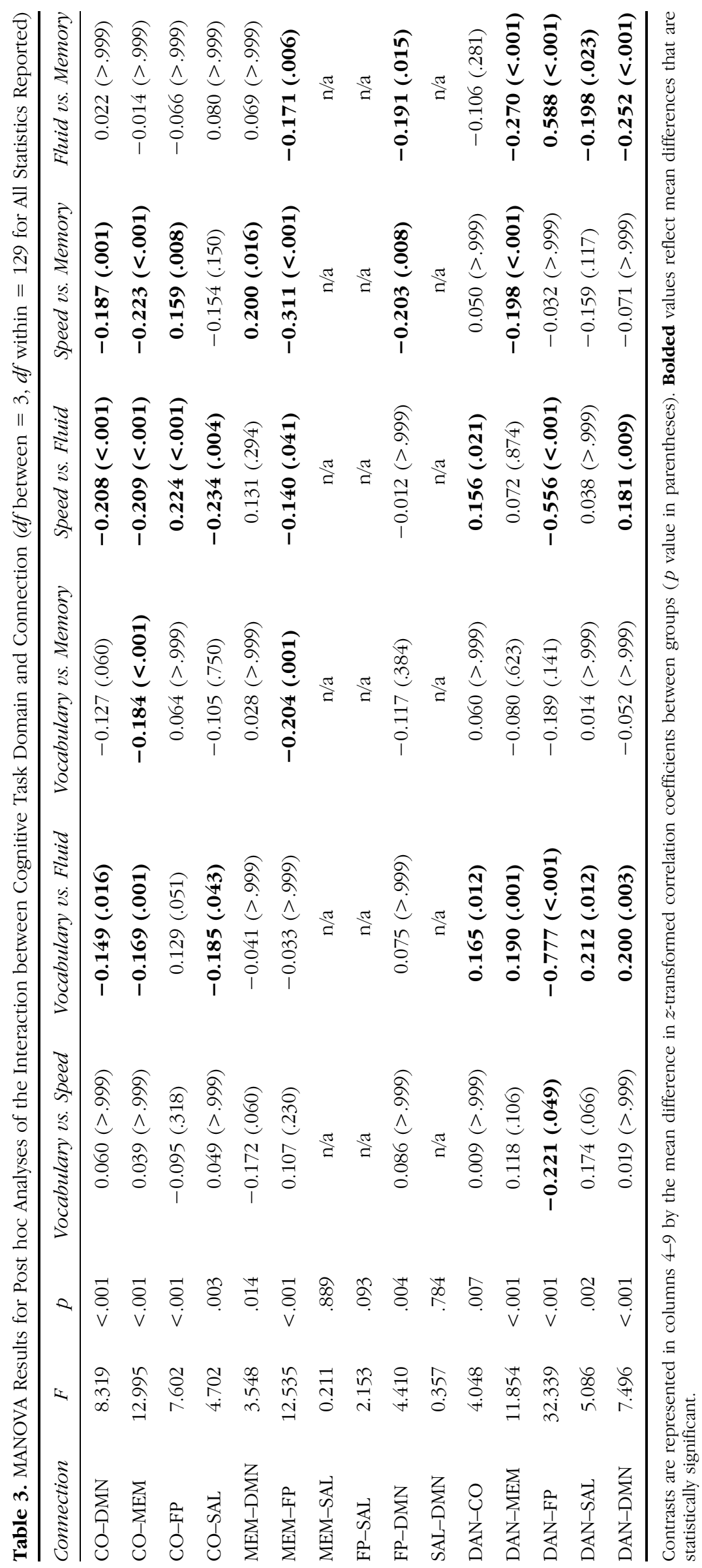


Table 4. Correlation Table for Spearman Correlation Coefficient ( $p$ Values in Parentheses) for the Relationship between Task Performance in Each Domain and Each Internetwork Connection

\begin{tabular}{|c|c|c|c|c|}
\hline & $V O C A B$ & SPEED & FLUID & MEM \\
\hline CO-DMN & $.033(.701)$ & $.022(.798)$ & $-.096(.263)$ & $-.004(.962)$ \\
\hline CO-MEM & $.078(.359)$ & $-.047(.587)$ & $-.070(.410)$ & $.024(.781)$ \\
\hline CO-FP & $-.021(.804)$ & $.086(.319)$ & $-.096(.259)$ & $.064(.459)$ \\
\hline CO-SAL & $.017(.841)$ & $-.152(.076)$ & $.073(.395)$ & $-.018(.835)$ \\
\hline MEM-DMN & $.048(.571)$ & $.070(.419)$ & $.229(.007)$ & $.212(.013)$ \\
\hline MEM-FP & $-.014(.869)$ & $.063(.465)$ & $-.171(.044)$ & $-.165(.053)$ \\
\hline MEM-SAL & $.172(.041)$ & $.090(.293)$ & $-.004(.963)$ & $.068(.429)$ \\
\hline FP-SAL & $-.139(.099)$ & $.085(.323)$ & $-.220(.009)$ & $-.040(.644)$ \\
\hline FP-DMN & $-.151(.074)$ & $.076(.379)$ & $-.189(.026)$ & $.056(.516)$ \\
\hline SAL-DMN & $.227(.007)$ & $-.014(.868)$ & $.156(.067)$ & $.055(.523)$ \\
\hline DAN-CO & $.120(.156)$ & $-.039(.654)$ & $-.001(.993)$ & $.019(.822)$ \\
\hline DAN-MEM & $.120(.158)$ & $-.093(.280)$ & $-.312(<.001)^{*}$ & $-.115(.178)$ \\
\hline DAN-FP & $.104(.218)$ & $.086(.319)$ & $.201(.018)$ & $-.050(.560)$ \\
\hline DAN-SAL & $-.075(.376)$ & $.042(.629)$ & $-.219(.010)$ & $.077(.370)$ \\
\hline DAN-DMN & $.062(.468)$ & $-.015(.862)$ & $-.177(.037)$ & $.114(.182)$ \\
\hline
\end{tabular}

Bolded correlation coefficients represent those that are significant at $p<.05$; correlation coefficients with an asterisk represent those that survive multiple comparison correction. VOCAB = Vocabulary; SPEED = Perceptual Speed; FLUID = Fluid Reasoning; MEM = Episodic Memory .

Table 5. Correlation Table for Spearman Correlation Coefficient ( $p$ Values in Parentheses) for the Relationship between Task Performance in Each Domain and Each Internetwork Connection after Controlling for Participant Age

\begin{tabular}{|c|c|c|c|c|}
\hline & $V O C A B$ & SPEED & FLUID & $M E M$ \\
\hline CO-DMN & $.009(.919)$ & $-.022(.802)$ & $-.100(.250)$ & $-.056(.521)$ \\
\hline CO-MEM & $.007(.934)$ & $-.131(.132)$ & $.004(.962)$ & $.080(.361)$ \\
\hline CO-FP & $.004(.961)$ & $.045(.609)$ & $-.033(.709)$ & $.110(.206)$ \\
\hline CO-SAL & $.078(.374)$ & $-.113(.194)$ & $-.003(.975)$ & $-.094(.282)$ \\
\hline MEM-DMN & $.075(.388)$ & $.175(.044)$ & .177 (.042) & $.102(.242)$ \\
\hline MEM-FP & $-.054(.540)$ & $-.080(.361)$ & $-.183(.035)$ & $-.094(.280)$ \\
\hline MEM-SAL & $.127(.146)$ & $.079(.365)$ & $.069(.431)$ & $.094(.282)$ \\
\hline FP-SAL & $-.164(.059)$ & $-.081(.352)$ & $-.163(.060)$ & $.038(.666)$ \\
\hline FP-DMN & $-.148(.088)$ & $-.036(.684)$ & $-.162(.062)$ & $.003(.975)$ \\
\hline SAL-DMN & $.251(.004)$ & $.114(.191)$ & $.124(.153)$ & $-.056(.523)$ \\
\hline $\mathrm{DAN}-\mathrm{CO}$ & $.131(.132)$ & $.050(.568)$ & $-.025(.777)$ & $-.024(.786)$ \\
\hline DAN-MEM & $.102(.244)$ & $-.069(.431)$ & $-.285(.001)^{*}$ & $-.064(.465)$ \\
\hline DAN-FP & $-.031(.726)$ & $.007(.937)$ & $.251(.004)$ & $-.036(.680)$ \\
\hline DAN-SAL & $-.086(.327)$ & $.056(.520)$ & $-.211(.015)$ & $.155(.075)$ \\
\hline DAN-DMN & $.062(.477)$ & $.052(.555)$ & $-.156(.073)$ & $.032(.712)$ \\
\hline
\end{tabular}

Bolded correlation coefficients represent those that are significant at $p<.05$; correlation coefficients with an asterisk represent those that survive multiple comparison correction. VOCAB = Vocabulary; SPEED = Perceptual Speed; FLUID = Fluid Reasoning; MEM = Episodic Memory . 


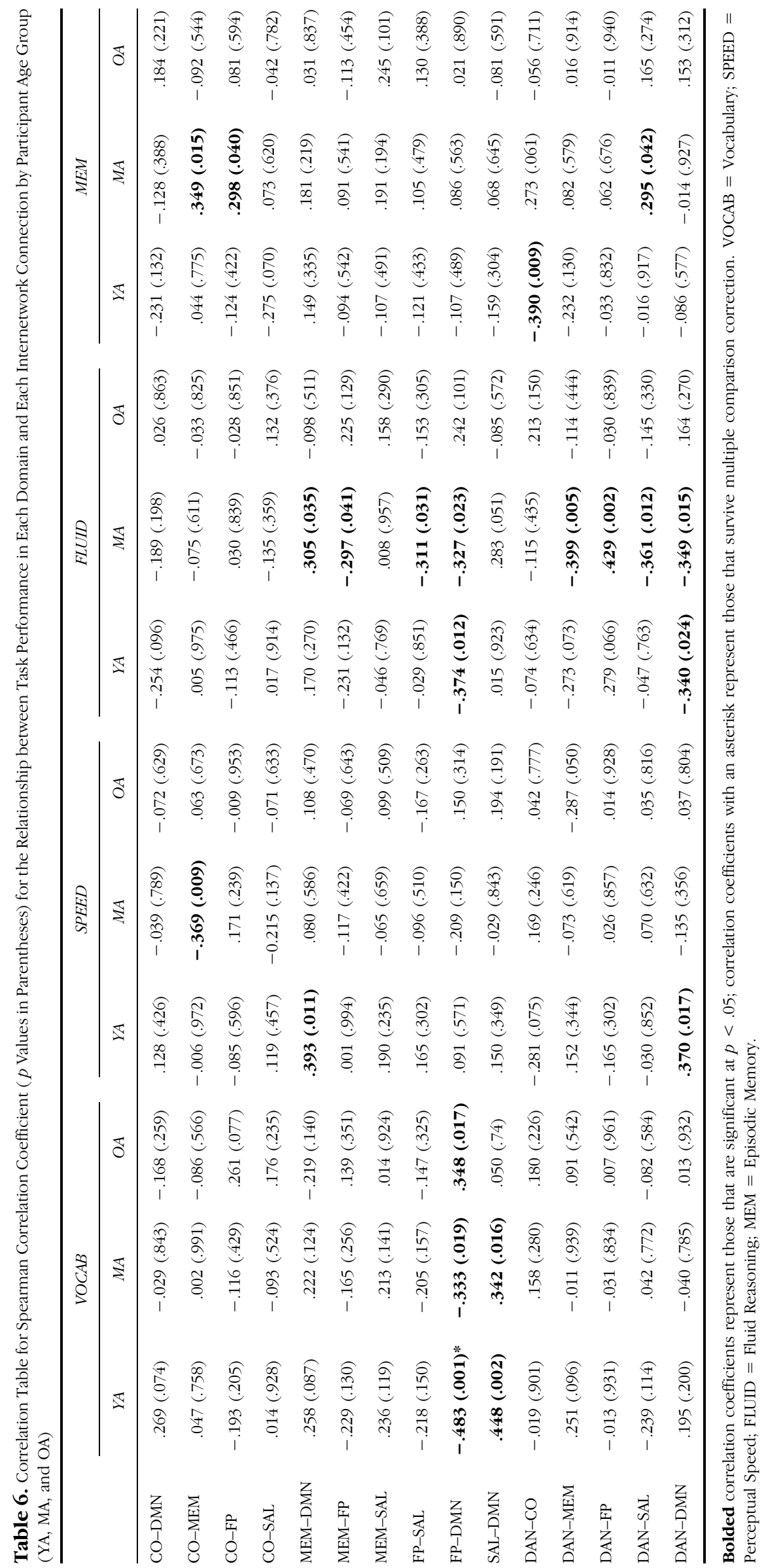




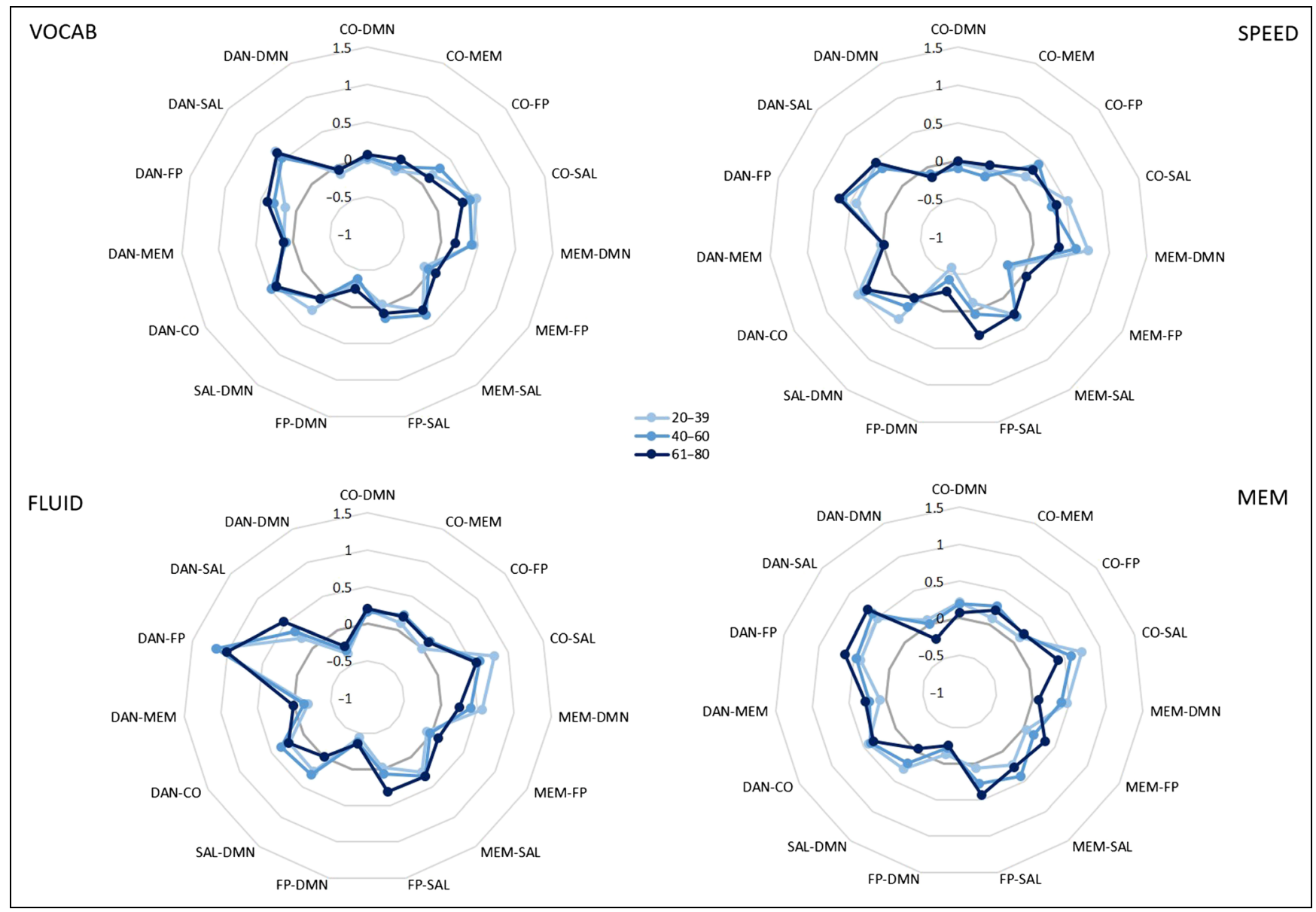

Figure 4. Between-network correlation coefficients for the nonsignificant interaction between age and connection within each domain.

That being said, when examining means for the connection by domain interaction within each age group, some interesting trends emerged, which may suggest that the effect of age on FP-DMN connectivity only exists during specific types of tasks (specifically, speed tasks). Although this three-way interaction was not significant, its $p$ value of .120 in the MANOVA analyses could suggest that we were simply underpowered to detect this complex effect (see Figure 4). Thus, although we failed to replicate that result here, it could be due to our inclusion of tasks tapping into multiple cognitive domains, rather than focusing on connectivity during one specific cognitive task. Specifically, if only the SPEED domain is modeled, there is a significant difference between older and younger adults in FP-DMN connectivity; however, given the scope of analyses in this study, we were unable to detect this small of an effect (see Figure 4, SPEED). As such, although this connection may be implicated in working memory, executive function, or processing speed tasks, it may not be a critically age-sensitive connection across all cognitive domains.

Furthermore, this study also did not provide evidence for a generally more connected brain in the context of aging. Specifically, the main effect of age was not significant, suggesting that, within this sample and during these tasks, OAs do not show overall greater connectivity across these networks relative to younger adults. Several past studies examining functional connectivity in the context of aging found that, generally speaking, OAs show more between-network connections than younger adults (King et al., 2018; Chan et al., 2014; Geerligs et al., 2014), a more positively connected brain graph (Ferreira et al., 2016), and reduced segregation/modularity of brain systems (Geerligs et al., 2015; Song et al., 2014). However, all but one (Geerligs et al., 2014) of these studies utilized resting-state fMRI data to probe such effects, and one of the studies similarly found patterns of internetwork connectivity that either increased or decreased with age (Geerligs et al., 2015). Thus, this study may not lie in contrast to these findings but may instead represent a novel approach for modeling and investigating the effect of age on internetwork connectivity.

We also found that connection strength between networks differed by the type of task being performed during the scan. From a broader perspective, these connectivity patterns appear to cluster such that certain tasks have more similar connectivity patterns between networks (i.e., vocabulary and speed, vocabulary and memory, and fluid and memory), whereas other tasks have largely dissimilar connectivity patterns between networks 
(i.e., speed and memory, speed and fluid, and vocabulary and fluid). This finding suggests that cognitive networks do modulate their connections to other networks based on cognitive task domain. Past studies on restingstate connectivity suggest its utility in revealing agerelated differences in network function, structure, and organization (Esposito et al., 2018; Damoiseaux, 2017; Hedden et al., 2016; Spreng et al., 2016; Dennis \& Thompson, 2014; Damoiseaux et al., 2008), although they may not fully represent how connectivity might change in the presence of a cognitive challenge. Although changes in network structure or activation were outside the scope of the present analyses, our results show how predefined, cognitively relevant networks may alter their relationships to each other in response to the demands of the task.

We also found that two internetwork connections showed a direct correlation with behavior: Greater connectivity between the DAN and the memory network was associated with poorer FLUID performance across the whole sample (and after controlling for participant age), and greater connectivity between the FP network and DMN was associated with poorer performance on VOCAB tasks. Although implicating different networks and tasks, these results somewhat mirror findings from previous studies showing differential connectivity patterns between older and younger adults that can be linked to differences in task performance (Prakash et al., 2012; Miller et al., 2008). Furthermore, one interesting trend evident in the correlational analyses conducted separately within each age group is the difference in the relationship between FP-DMN and VOCAB AND FLUID task performance based on age group. Although the negative relationship between FP-DMN connectivity and task performance was only significant for the VOCAB task in younger adults in the sample after correcting for multiple comparisons, the fact that this connection is positively related to VOCAB performance in OAs is a novel and unexpected finding (see Table 6). Although previous studies found that FP-DMN connectivity was consistently associated with poorer performance on executive function tasks (Damoiseaux, 2017; Prakash et al., 2012; Grady et al., 2010; Uddin et al., 2009), this study found a similar relationship with VOCAB task performance in younger adults (and marginally so with FLUID task performance) but found the opposite relationship with task performance in OAs. One potential effect that might influence this relationship is the superior performance on VOCAB tasks by OAs - it could be that these tasks are easier for OAs than they are for younger adults, and thus, this relationship between FP-DMN connectivity and task performance (frequently found to be a negative relationship in executive function tasks) simply reflects processing difficulty during the task. However, this finding of a positive relationship between FP-DMN connectivity and task performance in OAs was an unexpected finding in this study, and thus, interpretation of its meaning is purely speculative. By linking these connectivity patterns to task performance, this study adds to existing literature in identifying brain-based metrics that are directly linked to cognitive outcomes.

\section{Limitations and Future Directions}

One limitation of this study is the number of participants whose data were not usable in the context of the present analyses. Although the balanced design, stringent scrubbing criteria, and appropriateness of factor structure fit allowed for a robust set of data for analysis, they also resulted in significant data loss. That being said, one advantage of this method is that errors are produced if the predefined network structure is not an appropriate fit for the data. Although past studies have conducted analyses by parcellating correlation matrices based on predefined network organization, there is no validation as to whether these ROI network memberships are appropriate for each individual included in the analyses. This study, therefore, excluded roughly $54 \%$ of all valid data (sample size after scrubbing exclusion criteria $=312$; sample size of final analyses $=142$ ); however, the data that were included are data that we know fit the predefined network parcellation scheme. Had the analysis included these individuals (as it would have if we had examined connectivity using a standard correlational/graph theoretical approach), it would have included participants whose connectivity patterns may not have been appropriately modeled based on the predefined networks, thus potentially weakening the results. However, excluding these individuals also considerably reduced the power of the current study, which in turn might limit the generalizability of the findings presented here. Although this may somewhat limit the strength of the conclusions of this study, the within-participant design of the analyses somewhat assuages concerns about the strength of the effect of task domain on the observed connectivity patterns.

One additional aspect of the study that may have limited the strength of the results is the fact that an externally derived network parcellation scheme was utilized for defining network membership of ROIs in the present analyses (Power et al., 2011). This parcellation scheme was based on network organization at rest; however, the authors of the original study cross-validated its networks with task activation-based data to determine cognitive relevance of networks and to validate spatial distributions. As such, one strength of utilizing this parcellation system is that it was not derived based on the present data, and thus, the present results are not simply an artifact of double-dipping (Kriegeskorte, Simmons, Bellgowan, \& Baker, 2009). However, it is also possible that this network parcellation scheme may not represent an ideal fit for task-based connectivity data because it was derived during rest in an external sample or that it may not be appropriate for participants in this study as evidenced by the number of participants excluded for not exhibiting 
good model fit. Given that this study examined connectivity across a wide range of ages (20-80 years) and across four cognitive domains (VOCAB, SPEED, FLUID, and MEM), using an external network parcellation in the present analyses was crucial to avoid using a network parcellation that was biased by participant age or cognitive domain. Although it would have been possible to derive separate network parcellations for each cognitive domain in younger adults and then utilize these across all participants, this would have resulted in differential network structure for each cognitive domain (and, by definition, poorer fit for OAs), which would have made networkbased comparisons across the four domains inappropriate. Thus, although the results may have been slightly weakened by using this external network parcellation, it was necessary to appropriately test the hypotheses of this study.

Furthermore, a difference was found in VOCAB and FLUID task performance between those participants who were included and those who were excluded from the present analyses. Although task performance was not included as inclusion/exclusion criteria for these analyses, the subsample of individuals who met eligibility for these analyses tended to do better on these tasks. However, given that these included and excluded participants did not differ based on age, education, and NART IQ, this difference may not be representative of a systematic bias in the subsample of included participants.

Another limitation is the difference in NART IQ between older and younger/middle-aged participants. Although sampling was conducted in an unbiased, randomized way, this unequal distribution emerged, such that OAs tended to have higher verbal IQ than younger adults. This is consistent with previous studies reporting preserved or improved vocabulary/semantic memory in the context of healthy aging (Verhaeghen, 2003), which may be reflective of accrued verbal experience over adulthood, a cohort effect, or participation bias in that OAs with better cognitive functioning or more educational experience may be more likely to want to participate in cognitive aging studies, or some combination of these factors. Although these sources of bias are impossible to disentangle in this study, this may be a general limitation of most cross-sectional studies of cognitive aging.

\section{Conclusions}

Results from this study suggest that, in the context of aging, internetwork connectivity among a set of cognitive networks may be modulated by both age and cognitive process being employed. Furthermore, some of the differences in connectivity patterns based on age may also represent inefficient patterns of network recruitment, resulting in poorer behavioral performance as a result of this network inefficiency.

\section{Acknowledgments}

The authors thank Dr. Kathleen Gates at the University of North Carolina at Chapel Hill for her assistance with the MIIVsem analyses. This research was supported by a grant from the National Institute on Aging (R01 AG038465, PI Dr. Stern).

Reprint requests should be sent to Yaakov Stern, Cognitive Neuroscience Division, Department of Neurology, College of Physicians and Surgeons, Columbia University, 630 West 168th Street, P\&S Box 16, New York, NY 10032, or via e-mail: ys11@columbia.edu.

\section{REFERENCES}

Amer, T., Anderson, J. A. E., Campbell, K. L., Hasher, L., \& Grady, C. L. (2016). Age differences in the neural correlates of distraction regulation: A network interaction approach Neuroimage, 139, 231-239.

Avelar-Pereira, B., Bäckman, L., Wåhlin, A., Nyberg, L., \& Salami, A. (2017). Age-related differences in dynamic interactions among default mode, frontoparietal control, and dorsal attention networks during resting-state and interference resolution. Frontiers in Aging Neuroscience, 9, 152.

Birn, R. M., Diamond, J. B., Smith, M. A., \& Bandettini, P. A. (2006). Separating respiratory-variation-related fluctuations from neuronal-activity-related fluctuations in fMRI. Neuroimage, 31, 1536-1548.

Bollen, K. A. (1996). An alternative two stage least squares (2SLS) estimator for latent variable equations. Psychometrika, 61, 109-121.

Bollen, K. A. (2001). Two-stage least squares and latent variable models: Simultaneous estimation and robustness to misspecifications. In R. Cudeck, S. DuToit, \& D. Sörbom (Eds.), Structural equation modeling: Present and future (pp. 119-138). Lincolnwood, IL: Scientific Software.

Carp, J. (2013). Optimizing the order of operations for movement scrubbing: Comment on Power et al. Neuroimage, 76, 436-438.

Chan, M. Y., Alhazmi, F. H., Park, D. C., Savalia, N. K., \& Wig, G. S. (2017). Resting-state network topology differentiates task signals across the adult life span. Journal of Neuroscience, 37, 2734-2745.

Chan, M. Y., Park, D. C., Savalia, N. K., Petersen, S. E., \& Wig, G. S. (2014). Decreased segregation of brain systems across the healthy adult lifespan. Proceedings of the National Academy of Sciences, U.S.A., 111, E4997-E5006.

Chand, G. B., Wu, J., Hajjar, I., \& Qiu, D. (2017). Interactions of the salience network and its subsystems with the default-mode and the central-executive networks in normal aging and mild cognitive impairment. Brain Connectivity, 7, 401-412.

Dale, A. M., Fischl, B., \& Sereno, M. I. (1999). Cortical surfacebased analysis. I. Segmentation and surface reconstruction. Neuroimage, 9, 179-194.

Damoiseaux, J. S. (2017). Effects of aging on functional and structural brain connectivity. Neuroimage, 160, 32-40.

Damoiseaux, J. S., Beckmann, C. F., Arigita, E. J. S., Barkhof, F., Scheltens, P., Stam, C. J., et al. (2008). Reduced resting-state brain activity in the "default network" in normal aging. Cerebral Cortex, 18, 1856-1864.

Dennis, E. L., \& Thompson, P. M. (2014). Functional brain connectivity using fMRI in aging and Alzheimer's disease. Neuropsychology Review, 24, 49-62.

Dixon, M. L., Andrews-Hanna, J. R., Spreng, R. N., Irving, Z. C., Mills, C., Girn, M., et al. (2017). Interactions between the default network and dorsal attention network vary across default subsystems, time, and cognitive states. Neuroimage, 147, 632-649. 
Dosenbach, N. U. F., Fair, D. A., Miezin, F. M., Cohen, A. L., Wenger, K. K., Dosenbach, R. A. T., et al. (2007). Distinct brain networks for adaptive and stable task control in humans. Proceedings of the National Academy of Sciences, U.S.A., 104, 11073-11078.

Esposito, F., Bertolino, A., Scarabino, T., Latorre, V., Blasi, G., Popolizio, T., et al. (2006). Independent component model of the default-mode brain function: Assessing the impact of active thinking. Brain Research Bulletin, 70, 263-269.

Esposito, R., Cieri, F., Chiacchiaretta, P., Cera, N., Lauriola, M., Di Giannantonio, M., et al. (2018). Modifications in resting state functional anticorrelation between default mode network and dorsal attention network: Comparison among young adults, healthy elders and mild cognitive impairment patients. Brain Imaging and Behavior, 12, 127-141.

Ferreira, L. K., Regina, A. C. B., Kovacevic, N., Martin, M. G. M., Santos, P. P., Carneiro, C. G., et al. (2016). Aging effects on whole-brain functional connectivity in adults free of cognitive and psychiatric disorders. Cerebral Cortex, 26, 3851-3865.

Fischl, B., Salat, D. H., Busa, E., Albert, M., Dieterich, M., Haselgrove, C., et al. (2002). Whole brain segmentation: Automated labeling of neuroanatomical structures in the human brain. Neuron, 33, 341-355.

Fischl, B., van der Kouwe, A., Destrieux, C., Halgren, E., Ségonne, F., Salat, D. H., et al. (2004). Automatically parcellating the human cerebral cortex. Cerebral Cortex, 14, 11-22.

Fisher, Z. F., Bollen, K. A., \& Gates, K. M. (in press). A limited information estimator for dynamic factor models. Multivariate Behavioral Research. doi: 10.1080/ 00273171.2018 .1519406

Fisher, Z. F., Bollen, K. A., Gates, K. M., \& Rönkkö, M. (2017) MIIVsem: Model Implied Instrumental Variable (MIIV) estimation of structural equation models [Computer software manual]. Retrieved from https://CRAN.R-project.org/package= MIIVsem (R package version 0.5.2).

Fox, M. D., Snyder, A. Z., Vincent, J. L., Corbetta, M., Van Essen, D. C., \& Raichle, M. E. (2005). The human brain is intrinsically organized into dynamic, anticorrelated functional networks. Proceedings of the National Academy of Sciences, U.S.A., 102, 9673-9678.

Fujiyama, H., Van Soom, J., Rens, G., Gooijers, J., Leunissen, I., Levin, O., et al. (2016). Age-related changes in frontal network structural and functional connectivity in relation to bimanual movement control. Journal of Neuroscience, 36, 1808-1822.

Geerligs, L., Maurits, N. M., Renken, R. J., \& Lorist, M. M. (2014) Reduced specificity of functional connectivity in the aging brain during task performance. Human Brain Mapping, 35, 319-330.

Geerligs, L., Renken, R. J., Saliasi, E., Maurits, N. M., \& Lorist, M. M. (2015). A brain-wide study of age-related changes in functional connectivity. Cerebral Cortex, 25, 1987-1999.

Grady, C. L. (2008). Cognitive neuroscience of aging. Annals of the New York Academy of Sciences, 1124, 127-144.

Grady, C. L., Protzner, A. B., Kovacevic, N., Strother, S. C., Afshin-Pour, B., Wojtowicz, M., et al. (2010). A multivariate analysis of age-related differences in default mode and task-positive networks across multiple cognitive domains. Cerebral Cortex, 20, 1432-1447.

Grady, C. L., Sarraf, S., Saverino, C., \& Campbell, K. (2016). Age differences in the functional interactions among the default, frontoparietal control, and dorsal attention networks. Neurobiology of Aging, 41, 159-172.

Greicius, M. D., Supekar, K., Menon, V., \& Dougherty, R. F. (2009). Resting-state functional connectivity reflects structural connectivity in the default mode network. Cerebral Cortex, 19, 72-78.
Hedden, T., \& Gabrieli, J. D. E. (2004). Insights into the aging mind: A view from cognitive neuroscience. Nature Reviews Neuroscience, 5, 87-96.

Hedden, T., Schultz, A. P., Rieckmann, A., Mormino, E. C., Johnson, K. A., Sperling, R. A., et al. (2016). Multiple brain markers are linked to age-related variation in cognition. Cerebral Cortex, 26, 1388-1400.

Hirsiger, S., Koppelmans, V., Mérillat, S., Liem, F., Erdeniz, B., Seidler, R. D., et al. (2016). Structural and functional connectivity in healthy aging: Associations for cognition and motor behavior. Human Brain Mapping, 37, 855-867.

Jenkinson, M., Bannister, P., Brady, M., \& Smith, S. (2002). Improved optimization for the robust and accurate linear registration and motion correction of brain images. Neuroimage, 17, 825-841.

Jenkinson, M., Beckmann, C. F., Behrens, T. E. J., Woolrich, M. W., \& Smith, S. M. (2012). FSL. Neuroimage, 62, $782-790$

Jenkinson, M., \& Smith, S. (2001). A global optimisation method for robust affine registration of brain images. Medical Image Analysis, 5, 143-156.

Jones, D. T., Knopman, D. S., Gunter, J. L., Graff-Radford, J., Vemuri, P., Boeve, B. F., et al. (2016). Cascading network failure across the Alzheimer's disease spectrum. Brain, 139, 547-562.

King, B. R., van Ruitenbeek, P., Leunissen, I., Cuypers, K., Heise, K.-F., Santos Monteiro, T., et al. (2018). Age-related declines in motor performance are associated with decreased segregation of large-scale resting state brain networks. Cerebral Cortex, 28, 4390-4402.

Kriegeskorte, N., Simmons, W. K., Bellgowan, P. S. F., \& Baker, C. I. (2009). Circular analysis in systems neuroscience: The dangers of double dipping. Nature Neuroscience, 12, 535-540.

Laird, A. R., Eickhoff, S. B., Li, K., Robin, D. A., Glahn, D. C., \& Fox, P. T. (2009). Investigating the functional heterogeneity of the default mode network using coordinate-based meta-analytic modeling. Journal of Neuroscience, 29, 14496-14505.

Liu, J., Zhang, X., Yu, C., Duan, Y., Zhuo, J., Cui, Y., et al. (2016). Impaired parahippocampus connectivity in mild cognitive impairment and Alzheimer's disease. Journal of Alzheimer's Disease, 49, 1051-1064.

Miller, S. L., Celone, K., DePeau, K., Diamond, E., Dickerson, B. C., Rentz, D., et al. (2008). Age-related memory impairment associated with loss of parietal deactivation but preserved hippocampal activation. Proceedings of the National Academy of Sciences, U.S.A., 105, 2181-2186.

Parkes, L., Fulcher, B., Yücel, M., \& Fornito, A. (2018). An evaluation of the efficacy, reliability, and sensitivity of motion correction strategies for resting-state functional MRI. Neuroimage, 171, 415-436.

Power, J. D., Barnes, K. A., Snyder, A. Z., Schlaggar, B. L., \& Petersen, S. E. (2012). Spurious but systematic correlations in functional connectivity MRI networks arise from subject motion. Neuroimage, 59, 2142-2154.

Power, J. D., Cohen, A. L., Nelson, S. M., Wig, G. S., Barnes, K. A., Church, J. A., et al. (2011). Functional network organization of the human brain. Neuron, 72, 665-678.

Prakash, R. S., Heo, S., Voss, M. W., Patterson, B., \& Kramer, A. F. (2012). Age-related differences in cortical recruitment and suppression: Implications for cognitive performance. Behavioural Brain Research, 230, 192-200.

Razlighi, Q. R., Habeck, C., Barulli, D., \& Stern, Y. (2017). Cognitive neuroscience neuroimaging repository for the adult lifespan. Neuroimage, 144, 294-298.

Razlighi, Q. R., Habeck, C., Steffener, J., Gazes, Y., Zahodne, L. B., MacKay-Brandt, A., et al. (2014). Unilateral disruptions 
in the default network with aging in native space. Brain and Behavior, 4, 143-157.

Sala-Llonch, R., Peña-Gómez, C., Arenaza-Urquijo, E. M., VidalPiñeiro, D., Bargalló, N., Junqué, C., et al. (2012). Brain connectivity during resting state and subsequent working memory task predicts behavioural performance. Cortex, 48, 1187-1196.

Song, J., Birn, R. M., Boly, M., Meier, T. B., Nair, V. A., Meyerand, M. E., et al. (2014). Age-related reorganizational changes in modularity and functional connectivity of human brain networks. Brain Connectivity, 4, 662-676.

Spreng, R. N., Sepulcre, J., Turner, G. R., Stevens, W. D., \& Schacter, D. L. (2013). Intrinsic architecture underlying the relations among the default, dorsal attention, and frontoparietal control networks of the human brain. Journal of Cognitive Neuroscience, 25, 74-86.

Spreng, R. N., Stevens, W. D., Viviano, J. D., \& Schacter, D. L. (2016). Attenuated anticorrelation between the default and dorsal attention networks with aging: Evidence from task and rest. Neurobiology of Aging, 45, 149-160.

Stern, Y., Habeck, C., Steffener, J., Barulli, D., Gazes, Y., Razlighi, Q., et al. (2014). The Reference Ability Neural Network Study: Motivation, design, and initial feasibility analyses. Neuroimage, 103, 139-151.

Suckling, J., Simas, T., Chattopadhyay, S., Tait, R., Su, L., Williams, G., et al. (2015). A winding road: Alzheimer's disease increases circuitous functional connectivity pathways. Frontiers in Computational Neuroscience, 9, 140.

Uddin, L. Q., Kelly, A. M. C., Biswal, B. B., Castellanos, F. X., \& Milham, M. P. (2009). Functional connectivity of default mode network components: Correlation, anticorrelation, and causality. Human Brain Mapping, 30, 625-637.

Verhaeghen, P. (2003). Aging and vocabulary score: A meta-analysis. Psychology and Aging, 18, 332-339. 